\title{
A fine line between macrophage activation syndrome and multisystem inflammatory syndrome in children - literature review based on two case reports
}

\author{
Agnieszka Poniecka², Elżbieta Smolewska ${ }^{1,2}$ ID \\ ${ }^{1}$ Pediatric Center Maria Konopnicka, Central Clinical Hospital of the Medical University of Lodz, Poland \\ ${ }^{2}$ Department of Paediatric Cardiology and Rheumatology, Medical University of Lodz, Poland
}

\begin{abstract}
Multisystem inflammatory syndrome in children is a newly described condition temporarily associated with severe acute respiratory syndrome coronavirus 2. The primary observations indicated coronavirus disease 2019 infection in children to be mild. However, recent reports have revealed a correlation between multisystem inflammatory symptoms and coronavirus infection. The manifestation of the disease is similar to Kawasaki disease, toxic shock syndrome and macrophage activation syndrome. Treatment includes intravenous corticosteroids, immunoglobulin and biological therapy (anakinra, tocilizumab, infliximab).

In this article we compare world reports of multisystem inflammatory syndrome in children to case reports of two patients who were hospitalized recently at the Department of Paediatric Cardiology and Rheumatology. In the course of diagnostics they presented numerous symptoms which required differentiation from multisystem inflammatory syndrome in children.
\end{abstract}

Key words: paediatric multisystem inflammatory syndrome, SARS-CoV-2 infection, secondary haemophagocytic lymphohistiocytosis, macrophage activation syndrome.

\section{Introduction}

In late April 2020 when medical professionals from all over the world were concentrated on fighting coronavirus disease 2019 (COVID-19) a novel systemic disorder in children and adolescents named multisystem inflammatory syndrome in children (MIS-C)/paediatric inflammatory multisystem syndrome temporally associated with severe acute respiratory syndrome coronavirus 2 (SARS-CoV-2) infection (PIMS-TS) was described for the first time. It was initially observed by paediatricians in the United Kingdom (UK). Shortly afterwards, new cases were described in Italy and the United States of America (USA).

The first studies on the subject compared the symptoms of the novel syndrome to those observed in Kawasaki disease (KD), toxic shock syndrome (TSS), and secondary haemophagocytic lymphohistiocytosis/macrophage activation syndrome (SHLH/MAS) [1].
Based on the definition, macrophage activation syndrome (MAS) is described as continual activation and expansion of $\mathrm{T}$ lymphocytes and macrophages, which results in massive hypersecretion of proinflammatory cytokines leading to an overwhelming inflammatory reaction.

According to the European League Against Rheumatism, American College of Rheumatology and Paediatric Rheumatology International Trials Organisation collaborative initiative, MAS can be diagnosed in a febrile patient who meets the following criteria: ferritin > $685 \mathrm{ng} / \mathrm{ml}$ and any 2 of the following: decrease of platelet count mostly from thrombocytosis to $\leq 181 \times 10^{9} /$; aspartate aminotransferase > $48 \mathrm{U} /$; triglycerides > $156 \mathrm{mg} / \mathrm{dl}$, fibrinogen $\leq 360 \mathrm{mg} / \mathrm{dl}$. The criteria were established for patients with known or suspected systemic onset juvenile idiopathic arthritis (SOJIA) [2] (Fig. 1, Table I and Table II). 


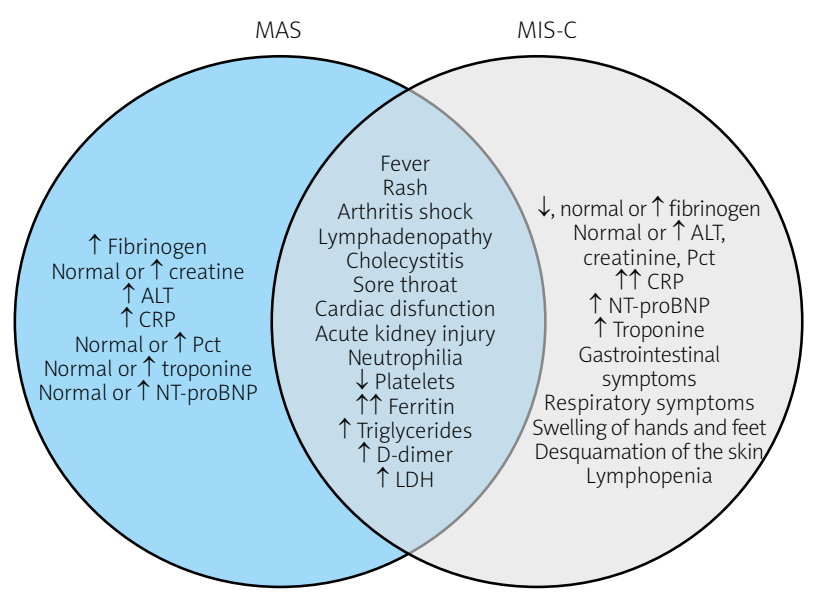

Fig. 1. List of symptoms and laboratory results common and specific to macrophage activation syndrome and multisystem inflammatory syndrome in children.

$\downarrow$-decreased, $\uparrow$ - increased $\uparrow \uparrow-$ highly increased. $A L T$ - alanine transaminase, AST - aspartate transaminase, $C K$ - creatine kinase, CK-MB mass - creatine kinase $M B$ isoform, $C R P-C$-reactive protein, ESR - erythrocyte sedimentation rate, GGTP - gamma-glutamyltransferase, INR - international normalized ratio, LDH-lactate dehydrogenase, MAS - macrophage activation syndrome, MIS-C - multisystem inflammatory syndrome in children, NT-proBNP - N-terminal prohormone of brain natriuretic peptide, Pct-procalcitonin, WBC-white blood cells.

According to the Centers for Disease Control and Prevention (CDC) MIS-C should be diagnosed in patients who fulfil the following criteria:

1. An individual aged $<21$ years with:

- fever $\geq 38.0^{\circ} \mathrm{C}$ lasting at least $24 \mathrm{~h}$,

- laboratory evidence of inflammation (one or more of the following: an elevated C-reactive protein (CRP), erythrocyte sedimentation rate (ESR), fibrinogen, procalcitonin (Pct), D-dimer, ferritin, lactate dehydrogenase (LDH), or interleukin 6 (IL-6); elevated neutrophils or reduced lymphocytes; low albumin),

- severe illness requiring hospitalization,

- evidence of involvement of two or more organ systems (i.e. cardiac, renal, respiratory, haematologic, gastrointestinal, dermatologic, neurological).

2. Laboratory confirmation of SARS-CoV-2 infection (positive SARS-CoV-2 testing by RT-PCR, serology, or antigen) or COVID-19 exposure within 4 weeks prior to onset of symptoms.

3. Exclusion of alternative diagnosis [3].

In this article we present the course of the disease and final diagnosis of two patients who were hospitalized recently at the Department of Paediatric Cardiology and Rheumatology.

Further, we describe the current knowledge about MIS-C/PIMS-TS, its pathogenesis, clinical symptoms and treatment, established based on cases reported principally from Europe and the USA.

Based on current understanding of MAS, the numerous symptoms presented by patients in the course of diagnostics in this report required differentiation from MIS-C. We further emphasize the difficulties in distinguishing MAS from MIS-C and the importance of gaining more data about MIS-C.

\section{Material and methods}

The objective of this article was to present the clinical problem of differentiation of MIS-C and MAS. We searched PubMed, ScienceDirect and Google Scholar databases using the following combinations of words: multisystem inflammatory syndrome in children (MIS-C), paediatric inflammatory multisystem syndrome temporally associated with SARS-CoV-2 infection (PIMSTS), pathogenesis and clinical symptoms of MIS-C, macrophage activation syndrome (MAS), secondary hemophagocytic lymphohistiocytosis (SHLH), Still's disease. The language was restricted to English. The discussion was based on the case study and a literature review.

\section{Results}

\section{Case 1}

A 17-year-old female patient was admitted in June 2020 to our department from the district hospital because of hectic fever, rash, joint pain, fatigue and elevated inflammatory markers which did not respond to the empiric antibiotic treatment. Based on the chest $X$-ray examination (CXR) she was also diagnosed with pneumonia. Apart from respiratory allergy (previously treated with systemic antihistamine drugs) she did not suffer from any chronic diseases.

On admission to the hospital, laboratory tests revealed significantly elevated CRP, ESR, D-dimer, ferritin, LDH, Pct, leukocytosis with immature form of leukocytes and mild anaemia (Table III). The RT-PCR test for COVID-19 was negative.

During hospitalization the general condition of the patient was fair. She suffered from hectic fever up to $39.5^{\circ} \mathrm{C}$, rash (Fig. 2), cough, peripheral joints, hands and feet pain, swelling of face, hands and feet, as well as pharyngitis and enlargement of the cervical lymph nodes.

Several imaging studies were conducted: ultrasound of the abdomen showed inflammation of gallbladder, high-resolution computed tomography (HRCT) revealed fibrous changes in the basal segments of the lungs and serial echocardiography (ECHO) revealed an increase in volume of pericardial fluid. Subsequent examination included bone marrow biopsy, which excluded lymphoproliferative disorder, and skin section of the rash, which 
Table I. List of symptoms and laboratory results common to patient 1, patient 2, macrophage activation syndrome and multisystem inflammatory syndrome in children

\begin{tabular}{|c|c|c|c|c|}
\hline Factor & Patient 1 & Patient 2 & MAS & MIS-C \\
\hline Fever & + & + & + & + \\
\hline Rash & + & + & + & + \\
\hline Arthritis & + & + & + & + \\
\hline Sore throat & + & + & + & + \\
\hline Lymphadenopathy & + & + & + & + \\
\hline Gastrointestinal symptoms & - & + & - & + \\
\hline Cholecystitis & + & + & + & + \\
\hline Respiratory symptoms & + & - & - & + \\
\hline Shock & - & + & + & ++ \\
\hline Acute kidney injury & - & + & + & + \\
\hline Cardiac dysfunction & - & + & + & + \\
\hline Swelling of hands and feet & + & + & - & + \\
\hline Desquamation of the skin & + & + & - & + \\
\hline WBC differential & $\begin{array}{l}\text { Neutrophilia, } \\
\text { lymphopenia }\end{array}$ & $\begin{array}{l}\text { Neutrophilia, } \\
\text { lymphopenia }\end{array}$ & Neutrophilia & $\begin{array}{l}\text { Neutrophilia, } \\
\text { lymphopenia }\end{array}$ \\
\hline Platelets & $\downarrow$ & $\downarrow \downarrow$ & $\downarrow$ & $\downarrow$ \\
\hline Ferritin & $\uparrow \uparrow$ & $\uparrow \uparrow$ & $\uparrow \uparrow$ & $\uparrow \uparrow$ \\
\hline Fibrinogen & $\downarrow$ & $\downarrow$ & $\downarrow$ & $\downarrow$, normal, or $\uparrow$ \\
\hline D-dimer & 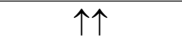 & 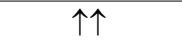 & $\uparrow$ & $\uparrow$ \\
\hline ALT & $\uparrow$ & $\uparrow \uparrow$ & $\uparrow$ & Normal, or $\uparrow$ \\
\hline Triglycerides & $\uparrow$ & 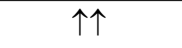 & $\uparrow$ & $\uparrow$ \\
\hline Creatinine & Normal & $\uparrow \uparrow$ & Normal or $\uparrow$ & $\uparrow$ \\
\hline CRP & $\uparrow \uparrow$ & $\uparrow \uparrow$ & $\uparrow$ & $\uparrow \uparrow$ \\
\hline Pct & $\uparrow$ & $\uparrow \uparrow$ & Normal or $\uparrow$ & $\uparrow$ \\
\hline NT-proBNP & NT & $\uparrow$ & Normal or $\uparrow$ & $\uparrow$ \\
\hline Troponin & NT & $\uparrow$ & Normal or $\uparrow$ & $\uparrow$ \\
\hline $\mathrm{LDH}$ & $\uparrow$ & $\uparrow$ & $\uparrow$ & $\uparrow$ \\
\hline
\end{tabular}

NT - not tested, $\uparrow$ - increased, $\uparrow \uparrow-$ highly increased, $\downarrow$ - decreased.

$A L T$ - alanine transaminase, $C R P-C$-reactive protein, $L D H$-lactate dehydrogenase, MAS - macrophage activation syndrome, MIS-C - multisystem inflammatory syndrome in children, NT-proBNP - N-terminal prohormone of brain natriuretic peptide, Pct-procalcitonin, WBC - white blood cells.

excluded systemic vasculitis. On the basis of several serology tests (including a COVID-19 antibody test) there was no confirmation of infectious cause of the disease.

Based on clinical symptoms (fever lasting $>1$ week, arthritis lasting $>2$ weeks, typical rash, lymphadenopathy and sore throat) as well as laboratory results (e.g. leukocytosis $>10,000 / \mu$ l with $>80 \%$ polymorphonuclear cells, abnormal liver function tests and negative test for rheumatoid factor) [2, 4], the patient was diagnosed with adult onset Still's disease (AOSD).

However, some other presented symptoms such as skin eruption in the area of the face, feet and hands preceding the swelling of the parts of the body, which are typical for different rheumatic diseases or fibrous changes in the basal segments of the lungs, led to the conclusion that MIS-C should be taken into account. However, neither the RT-PCR test nor serology confirmed COVID-19 infection (Table I).

Applied treatment included systemic glucocorticosteroids (the patient received a total of 5 pulses of methylprednisolone - $1.0 \mathrm{~g} /$ dose) and cyclosporine - $5 \mathrm{mg} / \mathrm{kg} /$ day (under control of drug concentration in the sera) with short-term partial clinical improvement.

However, in the following days relapse of fever, rash, adventitious sounds during auscultation of the lungs and general fatigue was observed. Furthermore, because 


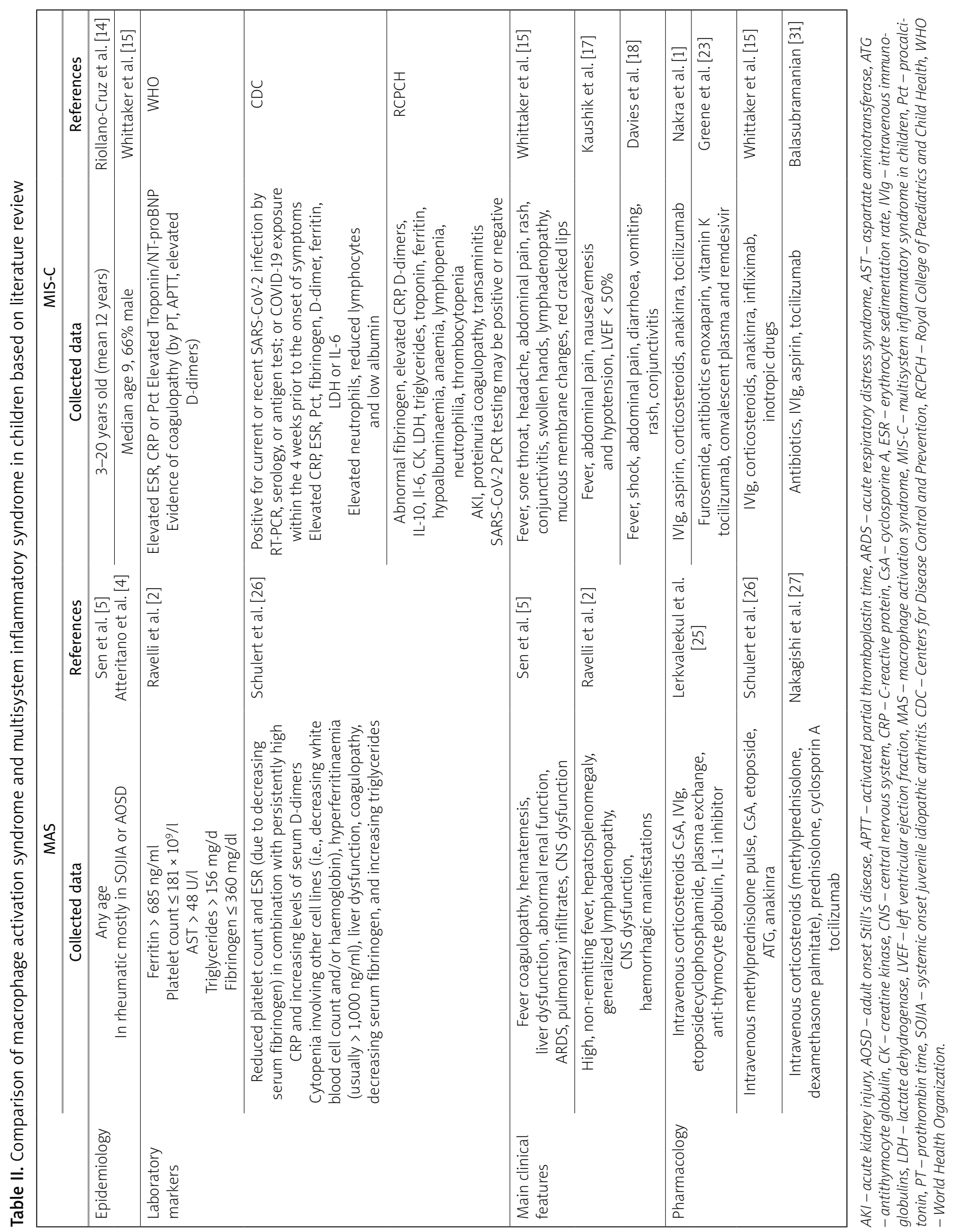


Table III. Case 1. Results of selected laboratory tests

\begin{tabular}{|c|c|c|c|c|}
\hline Parameter & $\begin{array}{l}\text { On admission } \\
\text { to the hospital }\end{array}$ & $\begin{array}{l}\text { Development } \\
\text { of MAS }\end{array}$ & $\begin{array}{l}\text { Last result before } \\
\text { discharging home }\end{array}$ & $\begin{array}{c}\text { Reference } \\
\text { normal range }\end{array}$ \\
\hline WBC $\left[10^{3} / \mu l\right]$ & 17.5 & 22.6 & 15.3 & $3.8-10$ \\
\hline Haemoglobin [g/dl] & 11 & 10.4 & 12 & $12.1-15.1$ \\
\hline Haematocrit [\%] & 34 & 31.7 & 37.1 & $35-44$ \\
\hline Platelets $\left[10^{3} / \mu \mathrm{l}\right]$ & 233 & 140 & 327 & $150-400$ \\
\hline Fibrinogen [mg/dl] & 344.32 & 308.23 & 300.12 & $200-400$ \\
\hline Ferritin $[\mu g / l]$ & 12014.52 & 10363.5 & 796.66 & $13-68$ \\
\hline D-dimer [ng/ml] & $>34836$ & $>34836$ & 724.32 & $0-500$ \\
\hline CRP $[\mathrm{mg} / \mathrm{l}]$ & 255.5 & 185.7 & 0.7 & $0-5$ \\
\hline $\operatorname{ESR}[\mathrm{mm} / \mathrm{h}]$ & 51 & 31 & 13 & $0-12$ \\
\hline Pct [ng/ml] & 0.78 & 1.58 & 0.05 & $<0.5$ \\
\hline Triglycerides [mg/dl] & 130 & 156 & 225 & $0-200$ \\
\hline ALT [U/l] & 24 & 657 & 27 & $0-33$ \\
\hline AST [U/I] & 86 & 1228 & 22 & $0-32$ \\
\hline GGTP [U/I] & 61 & 637 & 119 & $0-33$ \\
\hline LDH [U/I] & 820 & 3417 & 351 & $0-250$ \\
\hline
\end{tabular}

ALT - alanine transaminase, AST - aspartate transaminase, CRP - C-reactive protein, ESR - erythrocyte sedimentation rate, GGTP - gamma- glutamyltransferase, $L D H$ - lactate dehydrogenase, MAS - macrophage activation syndrome, Pct-procalcitonin, WBC-white blood cells.

of reported lower limb pain, swelling of subcutaneous and muscle tissue as well as fluid in ultrasound imaging, whole body nuclear magnetic resonance (NMR) was conducted. Inflammation of back fascia, lower and upper limbs, little fluid in the pleura and an increased signal in the thoracic vertebrae were revealed.

Due to subsequent dramatic deterioration of the clinical condition of the patient, persisting abnormalities in laboratory tests suggesting development of MAS (Table III), as well as lack of response to previous treatment, tocilizumab (8 mg/kg intravenous) was applied, with a good therapeutic effect.

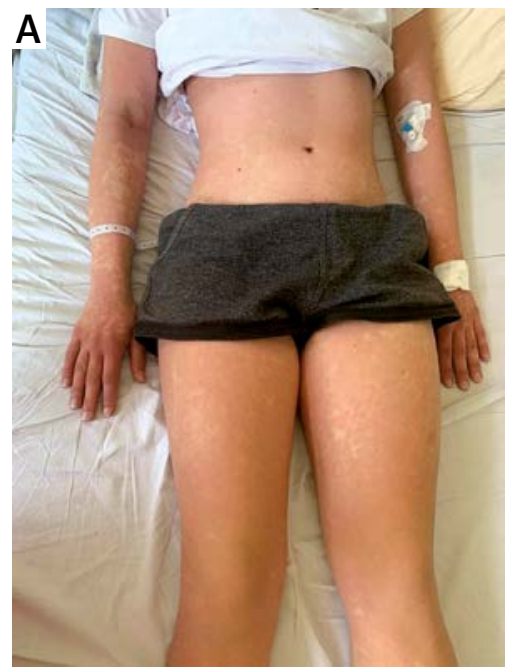

During hospitalization the patient received two doses of tocilizumab (a humanized anti-IL- 6 receptor antibody) with a two-week break in between. Treatment with cyclosporine and systemic glucocorticosteroids was continued. Gradual improvement of clinical state and laboratory test results was achieved (Table III). However, in the following days desquamation of the epidermis of the hands, feet and face was observed (Fig. 3).

The patient was discharged from the hospital on the $29^{\text {th }}$ day of hospitalization in a good general condition with the recommendation to continue current therapy under strict rheumatological control.

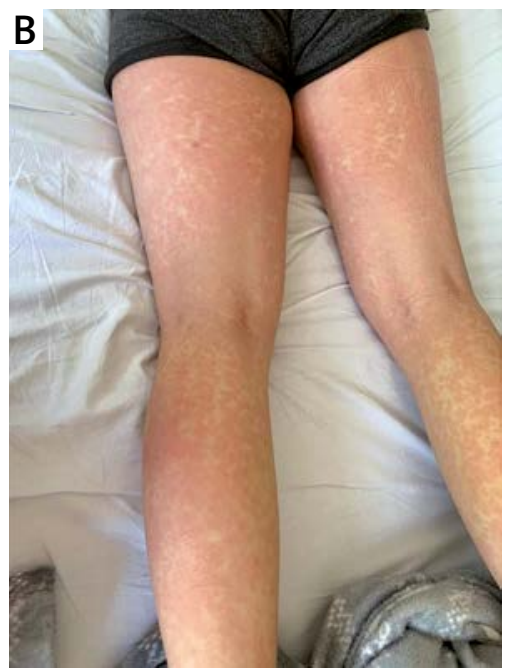

Fig. 2. Intensified salmon-pink macular rash, covering whole body, accompanied by painful swelling of hands. 

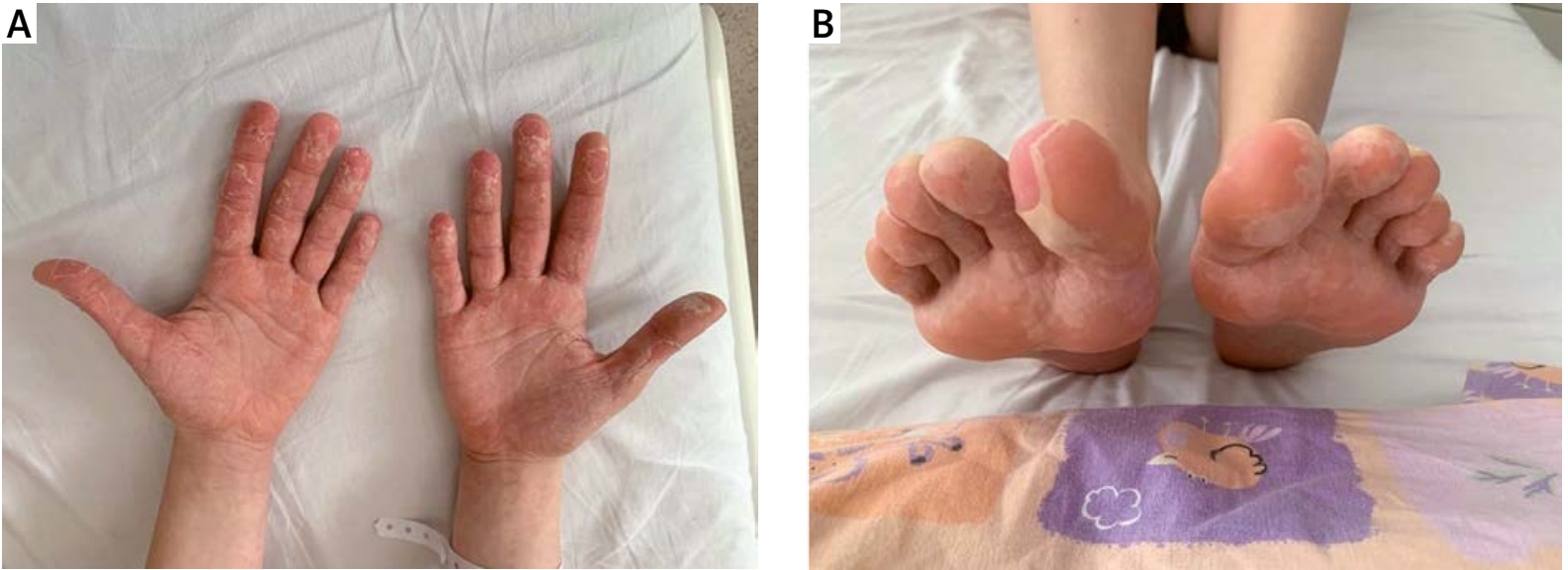

Fig. 3. Intensive desquamation of hands and feet skin which occurred after subacute phase.

In the following weeks of ambulatory observation, normalization of laboratory tests and health recovery were found. Apart from intensified hair loss the patient did not report any complaints.

\section{Case 2}

The 13-year-old female patient was admitted in June 2020 to our department because of fever reaching $38.2^{\circ} \mathrm{C}$ and rash lasting for 4 days. Moreover, the patient had suffered from symmetrical swelling of the ankles, knees and wrist joints for one week prior to other symptoms. Additionally, 4 months before admission to the hospital she was affected with a severe lower respiratory tract infection.

On admission to the hospital, physical examination revealed swelling of knee joints and pain of the thyroid gland on palpation. Laboratory tests revealed elevated inflammation markers and free thyroxine (FT4), accompanied by lower thyroid stimulating hormone (TSH).

According to her past medical history, she was diagnosed with Hashimoto disease 6 months prior to occurrence of the current symptoms and was treated with levothyroxine. On admission to the Emergency Department, the COVID-19 RT-PCR test was performed and gave a negative result. The following laboratory tests revealed significantly elevated C-reactive protein, ESR, D-dimer, ferritin, LDH, leukocytosis with immature forms and slightly elevated uric acid, aspartate aminotransferase (AST) and immunoglobulins in IgM and IgG class (Table IV).

Furthermore, immunological tests revealed clinically significant numbers (1 : 640 speckled) of anti-nuclear antibodies (ANA) and negative antibodies in ANA3 panel and anti-neutrophil cytoplasmic antibody (ANCA). Anticitrullinated protein antibodies (ACPA), rheumatoid factor (RF) and components $\mathrm{C} 3$ and $\mathrm{C} 4$ and total $\mathrm{CH} 50$ of the complement system were within the normal range.
Moreover, based on the positive enzyme-linked immunosorbent assay test (ELISA), confirmed in the western blot test, the patient was diagnosed with Lyme disease. Since the initial treatment consisted of $3^{\text {rd }}$ generation cephalosporin, the treatment was continued with a good clinical effect.

Ultrasound examination of peripheral joints showed slight inflammation of proximal and distal interphalangeal joints of both hands and metacarpophalangeal joints of fingers II-IV of both hands and the left wrist. There was no sign of inflammation in the other peripheral joints. The image of ECHO examination did not show any relevant abnormalities.

In the following days, the patient was in good condition. She reported slight joint pain, which was not accompanied with swelling, rash or fever. However, laboratory tests revealed persisting elevated markers of inflammation.

On the $10^{\text {th }}$ day of hospitalization the patient reported fever and strong pain of peripheral joints. The next day she suffered from itching of the vulva. Due to suspected urinary tract infection with a possible fungal co-infection, intravenous and local antifungal treatments as well as oral antibiotics were applied. On the following day the patient was fatigued. She suffered from diarrhoea, rash on the abdomen and lower limbs, hyperaesthesia, sore throat, pain and swelling of the vulva.

Laboratory tests revealed an extreme increase in inflammatory markers, aminotransaminases, uric acid, LDH, D-dimers, triglycerides, urea, creatinine, prolonged international normalized ratio (INR), hypoglycaemia and metabolic acidosis (Table IV).

Due to thrombocytopenia as well as possible neuroborreliosis, computed tomography of the head was performed, with no evidence of pathology. However, abdomen ultrasound showed acute kidney injury, acute cholecystitis and peritoneal fluid. In the next hours the 
Table IV. Case II. Results of selected laboratory tests

\begin{tabular}{|c|c|c|c|c|}
\hline Parameter & $\begin{array}{l}\text { On admission } \\
\text { to the hospital }\end{array}$ & $\begin{array}{l}\text { Development } \\
\text { of hypotonic shock } \\
\text { and MAS }\end{array}$ & $\begin{array}{l}\text { Last result before } \\
\text { discharging home }\end{array}$ & $\begin{array}{c}\text { Reference } \\
\text { normal range }\end{array}$ \\
\hline $\mathrm{WBC}\left[10^{3} / \mu \mathrm{l}\right]$ & 10.4 & 14.2 & 24.8 & $3.8-10.0$ \\
\hline Haemoglobin [g/dl] & 12 & 9.6 & 8.9 & $12.1-15.1$ \\
\hline Haematocrit [\%] & 35.7 & 29.2 & 26.8 & $35-44$ \\
\hline Platelets $\left[10^{3} / \mu \mathrm{l}\right]$ & 231 & 77 & 280 & $150-400$ \\
\hline Fibrinogen [mg/dl] & 405.36 & 264.38 & 228.72 & $200-400$ \\
\hline Ferritin $[\mu \mathrm{g} / \mathrm{l}]$ & 2239.93 & $>20000$ & 5558 & $13-68$ \\
\hline D-dimer [ng/ml] & 3937.68 & $>34836$ & 1115.23 & $0-500$ \\
\hline CRP $[\mathrm{mg} / \mathrm{l}]$ & 72.4 & 293.3 & 0.4 & $0-5$ \\
\hline $\mathrm{ESR}[\mathrm{mm} / \mathrm{h}]$ & 48 & 45 & 35 & $0-12$ \\
\hline Pct [ng/ml] & 0.18 & 67.79 & 0.08 & $<0.5$ \\
\hline Triglycerides [mg/dl] & 117 & 279 & 300 & $0-200$ \\
\hline ALT [U/l] & 9 & 69 & 432 & $0-33$ \\
\hline $\mathrm{AST}[\mathrm{U} / \mathrm{I}]$ & 34 & 380 & 109 & $0-32$ \\
\hline GGTP [U/I] & 11 & 190 & 1847 & $0-33$ \\
\hline $\mathrm{LDH}[\mathrm{U} / \mathrm{I}]$ & 368 & 4215 & 373 & $0-250$ \\
\hline Uric acid [mg/dl] & 7.2 & 15.5 & 7.2 & $2.4-5.7$ \\
\hline Urea [mg/dl] & 32 & 107 & 46 & $16.6-48.5$ \\
\hline Creatinine [mg/dl] & 0.72 & 4.84 & 0.72 & $0.52-0.79$ \\
\hline INR & 1.1 & 2.07 & 1.01 & $0.80-1.20$ \\
\hline Glucose [mg/dl] & 86 & 33 & 115 & 70-99 \\
\hline CK [U/I] & - & 591 & - & $29-168$ \\
\hline CK-MB mass [mg/dl] & - & 8.8 & 0.5 & $0-3.1$ \\
\hline Troponin I [mg/dl] & - & 0.62 & - & $<0.028$ \\
\hline NT-proBNP [pg/ml] & - & 4183 & 541.2 & $0-125$ \\
\hline
\end{tabular}

ALT - alanine transaminase, AST - aspartate transaminase, $C K$-creatine kinase, CK-MB mass-creatine kinase $M B$ isoform, CRP-C-reactive protein, ESR - erythrocyte sedimentation rate, GGTP - gamma-glutamyltransferase, INR - international normalized ratio, LDH - lactate dehydrogenase, MAS - macrophage activation syndrome, NT-proBNP-N-terminal prohormone of brain natriuretic peptide, Pct-procalcitonin, WBC - white blood cells.

patient presented multiple organ dysfunction syndrome with hypotonic shock and disseminated intravascular coagulation (DIC).

Hospitalization in the intensive care unit was needed. Furthermore, subsequent laboratory tests revealed an increase of cardiac troponin I (cTnl), N-terminal prohormone of brain natriuretic peptide (NT-proBNP), creatine kinase $(C K)$ and creatine kinase $M B$ isoform (CK-MB mass). Widespread antibiotic and intravenous antifungal and catecholamine treatments were applied.

Because of suspected MAS developed from connective tissue disease, after exclusion of lymphoproliferative disorder, the patient received an infusion of methylprednisolone (initially in a single dose of $1.0 \mathrm{~g}$ and then $2 \mathrm{mg} / \mathrm{kg} / \mathrm{h}$ ), cyclosporine (in a dose of $5 \mathrm{mg} / \mathrm{kg}$ ) and intravenous immunoglobulins (IVIg), with no clinical effect. The patient required mechanical ventilation, further catecholamine supply and multiple blood transfusion.

Due to her critical condition, the patient received tocilizumab (8 $\mathrm{mg} / \mathrm{kg}$ intravenously) with gradual clinical improvement. During hospitalization in the intensive care unit, ulcers as well as microbial and bleeding blisters on the mouth were noted. Moreover, a massive desquamation of whole body epidermis was observed, which could not be related to any previous treatment. The subsequent diagnostic examinations included lumbar puncture which did not reveal the cause of infection.

Therapy by systemic glucocorticosteroids, cyclosporine, tocilizumab infusion (with a two-week break in between) and wide spectrum antibiotics and antifungals 
was continued with slow clinical and laboratory improvement (Table IV). The patient was finally diagnosed with MAS, shock syndrome, acute kidney injury and acute liver failure with the recommendation of further observation for connective tissue disease. On the $39^{\text {th }}$ day of hospitalization the patient was discharged home in fairly good, stable general condition with the recommendation to continue current therapy.

The patient was admitted to the hospital every two weeks (followed by further observation every month) for physical and laboratory checks and to receive intravenous infusion of tocilizumab. An increase in muscle strength, wound healing and improvement of kidney function were achieved. However, the increase of aminotransferases and hepatic steatosis in abdomen ultrasound has persisted up to the present time.

\section{Discussion}

Macrophage activation syndrome is a severe complication of a number of rheumatic diseases which can lead to multi-organ dysfunction or even death in 8 to $22 \%$ of cases [5]. It may develop at any age. In rheumatic disorders it is most commonly featured in SOJIA or AOSD [4]. However, it is also reported in other autoimmune or autoinflammatory conditions, i.e. adult- and childhood-onset systemic lupus erythematosus, KD, and periodic fever syndromes.

Typical clinical manifestations of MAS include persistent fever, altered mental state, lymphadenopathy and hepatosplenomegaly. The later stages of the disease lead to haemorrhagic, disseminated intravascular coagulation (DIC).

In many reports renal involvement has also been described. The data indicate a substantially higher risk of mortality in patients with renal dysfunction $[5,6]$. Late recognition of symptoms and initiation of intensive treatment may cause hospitalization in an intensive care unit or even death.

In the first case the patient was diagnosed with AOSD based on clinical symptoms (fever lasting $>1$ week, arthritis lasting > 2 weeks, typical rash, lymphadenopathy and sore throat) as well as laboratory results (e.g. leukocytosis > 10,000/ $\mu$ l with $>80 \%$ polymorphonuclear cells, abnormal liver function tests and a negative test for rheumatoid factor) [7, 8] (Table II).

Some other presented symptoms such as skin eruption in the area of the face, feet and hands preceding the swelling of the parts of the body, which are typical for different rheumatic diseases, or fibrous changes in the basal segments of the lungs led to the conclusion that MIS-C should be taken into account. However, neither the RT-PCR test nor serology confirmed COVID-19 infection (Table I).
The cause of development of MAS in the second case was ambiguous. The initial diagnosis of Lyme disease and applied treatment appeared to be correct. The patient responded with clinical improvement (no reported fever or rash and reduction of joint pain) to the therapy.

However, a sudden dramatic exacerbation of clinical condition, and multi-organ failure, revealed by laboratory tests, suggested that many other conditions such as SOJIA, TSS or neuroborreliosis should be taken into consideration.

Moreover, a few laboratory results ruled out other potential conditions (e.g. sterile culture of blood and cerebrospinal fluid). The coexistence of further symptoms such as diarrhoea, sore throat, hyperaesthesia, sudden hypotonic shock and massive desquamation is not typical for any previously known rheumatic syndromes. Therefore, MIS-C was suggested as the cause of the observed clinical state (Table I).

Multisystem inflammatory syndrome in children is a novel syndrome currently associated with SARS-CoV-2 infection. A multisystem inflammatory condition with some features similar to those of KD, MAS and TSS was first reported in the UK, Italy and the USA.

The definitions announced by the World Health Organization (WHO) [9] and the Royal College of Paediatrics and Child Health (RCPCH) are similar to CDC, although RCPCH allows the possibility of a negative SARS-CoV-2 RT-PCR test and does not refer to serology, antigen or COVID-19 exposure [10]. Most registries include only patients with confirmed SARS-CoV-2 infection.

However, some case reports include children with hyperinflammatory shock with no confirmed evidence of SARS-CoV-2 [11, 12]. According to the definition, MIS-C may be diagnosed in patients under the age of 21 $[3,9,10]$ in all racial groups. However, a higher proportion of African or Hispanic children is observed [13]. The median age is 12 and it can happen more often in males [14] (Table II).

Although RT-PCR and serology did not confirm COVID-19 infection in our patients, it should be mentioned that the patients had RT-PCR only on admission and in the second case the diagnostic serology was performed after administration of IVIg, which could have affected the test result.

Recent reports associate other new symptoms with MIS-C. Although they are not specific to one syndrome, it appears that MIS-C connects manifestation of KD, MAS and TSS in one disease (Fig. 1, Table I).

Fever for more than 24 hours [3] or 3 days [10] accompanied with a rash is the primary described prodrome, which also applied to our patients' admission to the hospital. The observations show that almost all reported cases have a history of fever [15-18]. 
Typically, gastrointestinal presentation such as abdominal pain, nausea, vomiting or diarrhoea is also observed $[19,20]$. Subsequent described symptoms are sore throat, lymphadenopathy and swollen hands and feet (Case 1) [15]. Manifestations of the respiratory system, like those observed in case 1 , are common.

A serious complication is cardiac involvement. The most common manifestations in MIS-C are reduced LVEF $<55 \%$, pericardial effusion or coronary arteries dilatation or aneurysm [20]. Likewise, a more severe spectrum of disease with a tendency to multiorgan involvement, hypovolaemia and shock which often requires hospitalization in an intensive care unit is more common in MIS-C [18, 21-23] (Table II).

According to observations in the USA the majority of patients diagnosed with MIS-C had symptoms involving at least four organ systems [24], from which the most commonly involved were the gastrointestinal, cardiovascular, haematological, mucocutaneous and respiratory systems.

Pharmacological treatment of MAS consists of parenteral administration of high doses of glucocorticosteroids, which are slowly decreased over the following days if the patient responds well. If the improvement is not satisfactory, additional therapy with cyclosporine is recommended. Common side effects include hypertension and renal toxicity. Etoposide, anti-thymocyte globulin (ATG), IVIg, cyclophosphamide, and plasma exchange are also mentioned as treatment options.

However, because of severe side effects such as severe bone marrow suppression, hepatic and renal impairment (etoposide), increased risk of severe infection and mortality (ATG) or inconsistent outcomes (IVIg, cyclophosphamide, and plasma exchange), they are not recommended as a first-line therapy for MAS. Biological therapies for MAS have become commonly used. Because of the fact that interleukin 1 (IL-1) and IL-6 play a key role in the pathogenesis of SOJIA, the use of the IL-1 receptor antagonist anakinra has been reported to benefit patients with an MAS-associated SOJIA condition.

However, there are a few reports that anakinra may also be a trigger for MAS. Subsequent therapies tested included supply of the anti-IL-1 $\beta$ monoclonal antibody canakinumab. However, according to case reports, the development of MAS in SOJIA patients treated with canakinumab was not statistically significantly different from that in placebo-treated patients. Administration of the anti-IL-6 receptor monoclonal antibody tocilizumab had promising results, but the data on the treatment of an MAS-associated SOJIA condition are limited [25-27] (Table II).

Because SARS-CoV-2 is not usually detected in patients with MIS-C, the recent reports suggest that it is the acquired immune response, rather than increased viral replication, that leads to the antibody-dependent enhancement of inflammation. Although the pathology of MIS-C is still not defined, it is highly possible that the antibodies to SARS-CoV produced after previous SARS-CoV-2 infection cause the formation of immune complexes or direct anti-tissue antibody activation or cellular activation, which leads to triggering the host inflammatory response [13].

Based on previous observations of the clinical course of MIS-C [28-30], as well as a satisfactory treatment trial of COVID-19 infection with immunomodulatory drugs [28-30], it is believed that children diagnosed with MIS-C should respond well to aggressive immunomodulatory and anti-inflammatory therapies.

Although there have not been enough studies comparing the clinical efficacy of various treatment options in MIS-C, the treatment has consisted primarily of supportive care and directed therapy against the underlying inflammatory process. The use of glucocorticosteroids, IVIg, cyclosporine as well as anakinra and tocilizumab has proven to be successful [1, 15, 23, 31]. In some clinical states (e.g. low platelet, coronary artery aneurysms) therapy should be supported with low-dose aspirin [32, 33] (Table I).

In the presented cases intravenous glucocorticosteroids, cyclosporine and IVIg were implemented based on the previous reports. However, the clinical effect was not satisfactory and it was decided that addition of biological treatment (tocilizumab) was necessary.

\section{Conclusions}

Multisystem inflammatory syndrome in children is a new disease associated with SARS-CoV-2 infection which is similar to MAS in clinical presentation, laboratory test results and applied treatment. An increasing number of reported cases from around the world provide characterization of typical symptoms.

However, many aspects such as diagnostic criteria, treatment or pathogenesis are yet to be determined. Although RT-PCR and serology in both presented cases did not confirm COVID-19 infection, the clinical courses in both situations were not typical for any known diseases or systemic symptoms, suggesting that MIS-C could be diagnosed.

Further research on pathogenesis and detailed diagnostic criteria for MIS-C are needed. It seems to be a new diagnostic and therapeutic challenge in the COVID-19 pandemic period.

The authors declare no conflict of interest. 


\section{References}

1. Nakra NA, Blumberg DA, Herrera-Guerra A, Lakshminrusimha S. Multi-system inflammatory syndrome in children (MIS-C) following SARS-CoV-2 infection: review of clinical presentation, hypothetical pathogenesis, and proposed management Children (Basel) 2020; 7: 69, DOI: 10.3390/children7070069.

2. Ravelli A, Minoia F, Davì S, et al. 2016 classification criteria for macrophage activation syndrome complicating systemic juvenile idiopathic arthritis: a European League Against Rheumatism/American College of Rheumatology/Paediatric Rheumatology International Trials Organisation collaborative initiative. Arthritis Rheumatol 2016; 68: 566-576, DOI: 10.1002/ art.39332.

3. CDC Health Alert Network. Multisystem inflammatory syndrome in children (MIS-C) associated with coronavirus disease 2019 (COVID-19). https://emergency.cdc.gov/han/2020/ han00432.asp [Accessed: 23.05.2020].

4. Atteritano M, David A, Bagnato G, et al. Haemophagocytic syndrome in rheumatic patients. A systematic review. Eur Rev Med Pharmacol Sci 2012; 16: 1414-1424.

5. Sen ES, Clarke SL, Ramanan AV. Macrophage activation syndrome. Indian J Pediatr 2016; 83: 248-253, DOI: 10.1007/s12098015-1877-1.

6. Ramanan AV, Rosenblum ND, Feldman BM, et al. Favourable outcome in patients with renal involvement complicating macrophage activation syndrome in systemic onset juvenile rheumatoid arthritis. J Rheumatol 2004; 31: 2068-2070.

7. Efthimiou P, Paik PK, Bielory L. Diagnosis and management of adult onset Still's disease. Ann Rheum Dis 2006; 65: 564-572, DOI: 10.1136/ard.2005.042143.

8. Jain D, Aggarwal HK, Rao A, et al. Macrophage activation syndrome in a patient with systemic onset of the juvenile idiopathic arthritis. Reumatologia 2016; 54: 42-47, DOI: 10.5114/ reum.2016.58763.

9. https://www.who.int/news-room/commentaries/detail/multisystem-inflammatory-syndrome-in-children-and-adolescentswith-covid-19.

10. https://www.rcpch.ac.uk/resources/guidance-paediatric-multisystem-inflammatory-syndrome-temporally-associatedcovid-19-pims.

11. Riphagen S, Gomez X, Gonzalez-Martinez C, et at. Hyperinflammatory shock in children during COVID-19 pandemic. Lancet 2020; 395: 1607-1608, DOI: 10.1016/S0140-6736(20)31094-1.

12. Verdoni L, Mazza A, Gervasoni A, et al. An outbreak of severe Kawasaki-like disease at the Italian epicentre of the SARSCoV-2 epidemic: an observational cohort study. Lancet 2020; 395: 1771-1778, DOI: 10.1016/S0140-6736(20)31103-X.

13. Jiang L, Tang K, Levin M, et al. COVID-19 and multisystem inflammatory syndrome in children and adolescents. Lancet Infect Dis 2020; 20: e276-e288, DOI: 10.1016/S1473-3099 (20)30651-4

14. Riollano-Cruz M, Akkoyun E, Briceno-Brito E, et al. Multisystem inflammatory syndrome in children related to COVID-19: a New York City experience. J Med Virol 2020, DOI: 10.1002/ jmv.26224 [Online ahead of print].

15. Whittaker E, Bamford A, Kenny J, et al. Clinical characteristics of 58 children with a pediatric inflammatory multisystem syn- drome temporally associated with SARS-CoV-2. JAMA 2020; 324: 259-269, DOI: 10.1001/jama.2020.10369.

16. Verdoni L, Mazza A, Gervasoni A, et al. An outbreak of severe Kawasaki-like disease at the Italian epicentre of the SARSCoV-2 epidemic: an observational cohort study. Lancet 2020; 395: 1771-1778, DOI: 10.1016/S0140-6736(20)31103-X.

17. Kaushik S, Aydin SI, Derespina KR, et al. Multisystem inflammatory syndrome in children associated with severe acute respiratory syndrome coronavirus 2 infection (MIS-C): a multi-institutional study from New York City. J Pediatr 2020; 224: 24-29, DOI: 10.1016/j.jpeds.2020.06.045.

18. Davies P, Evans C, Kanthimathinathan HK, et al. Intensive care admissions of children with paediatric inflammatory multisystem syndrome temporally associated with SARS-CoV-2 (PIMS-TS) in the UK: a multicentre observational study. Lancet Child Adolesc Health 2020; 4: 669-677, DOI: 10.1016/S23524642(20)30215-7.

19. Radia T, Williams N, Agrawal P, et al. Multi-system inflammatory syndrome in children \& adolescents (MIS-C): a systematic review of clinical features and presentation. Paediatr Respir Rev 2020, DOI: 10.1016/j.prrv.2020.08.001 [Online ahead of print].

20. Lawrensia S, Henrina J, Wijaya E, et al. Pediatric inflammatory multisystem syndrome temporally associated with SARSCoV-2: a new challenge amid the pandemic. SN Compr Clin Med 2020, DOI: 10.1007/s42399-020-00602-8 [Online ahead of print].

21. Nijman RG, De Guchtenaere A, Koletzko B, et al. Pediatric inflammatory multisystem syndrome: statement by the Pediatric Section of the European Society for Emergency Medicine and European Academy of Pediatrics. Front Pediatr 2020; 8: 490, DOI: 10.3389/fped.2020.00490.

22. Chiotos K, Bassiri H, Behrens EM, et al. Multisystem inflammatory syndrome in children during the coronavirus 2019 pandemic: a case series. J Pediatric Infect Dis Soc 2020; 9: 393-398, DOI: 10.1093/jpids/piaa069.

23. Greene AG, Saleh M, Roseman E, Sinert R. Toxic shock-like syndrome and COVID-19: A case report of multisystem inflammatory syndrome in children (MIS-C). Am J Emerg Med 2020; 38: 2492.e5-2492.e6, DOI: 10.1016/j.ajem.2020.05.117.

24. Feldstein LR, Rose EB, Horwitz SM, et al. Multisystem inflammatory syndrome in U.S. children and adolescents. N Engl I Med 2020; 383: 334-346, DOI: 10.1056/NEJMoa2021680.

25. Lerkvaleekul B, Vilaiyuk S. Macrophage activation syndrome: early diagnosis is key. Open Access Rheumatol 2018; 10: 117-128, DOI: 10.2147/OARRR.S151013.

26. Schulert GS, Grom AA. Pathogenesis of macrophage activation syndrome and potential for cytokine-directed therapies. Annu Rev Med 2015; 66: 145-159, DOI: 10.1146/annurev-med061813-012806.

27. Nakagishi Y, Shimizu M, Kasai K, et al. Successful therapy of macrophage activation syndrome with dexamethasone palmitate. Mod Rheumatol 2016; 26: 617-620, DOI: 10.3109/ 14397595.2014.906053.

28. Consiglio CR, Cotugno N, Sardh F, et al. The Immunology of multisystem inflammatory syndrome in children with COVID-19. Cell 2020; 183: 968-981.e7, DOI: 10.1016/j.cell.2020.09.016. 
29. Opoka-Winiarska V, Grywalska E, Roliński J. Could hemophagocytic lymphohistiocytosis be the core issue of severe COVID-19 cases? BMC Med 2020; 18: 214, DOI: 10.1186/s12916020-01682-y.

30. Leszczyński P. COVID-19: a short message to rheumatologists. Reumatologia 2020; 58: 130-133, DOI: 10.5114/ reum.2020.96685.

31. Balasubramanian S, Nagendran TM, Ramachandran B, Ramanan AV. Hyper-inflammatory syndrome in a child with COVID-19 treated successfully with intravenous immunoglobulin and tocilizumab. Indian Pediatr 2020; 57: 681-683, DOI: 10.1007/s13312-020-1901-z.
32. Henderson LA, Canna SW, Friedman KG, et al. American College of Rheumatology clinical guidance for multisystem inflammatory syndrome in children associated with SARS-CoV-2 and hyperinflammation in pediatric COVID-19: version 1. Arthritis Rheumatol 2020, DOI: 10.1002/art.41454 [Online ahead of print].

33. Gupta A, Gill A, Sharma M, Garg M. Multi-system inflammatory syndrome in a child mimicking Kawasaki disease. J Trop Pediatr 2020, DOI: 10.1093/tropej/fmaa060 [Online ahead of print]. 